\title{
Simulación genérica a nivel de sistema para soluciones avanzadas de gestión de recursos
}

\author{
Paula Rodríguez, Paula Sarasúa, Luis Diez, Ramón Agüero \\ Departamento de Ingeniería de Comunicaciones \\ Universidad de Cantabria \\ Plaza de la Ciencia, s/n. 39005 Santander \\ \{paula.rodriguez, paula.sarasua\}@alumnos.unican.es, \{ldiez, ramon\}@tlmat.unican.es
}

\begin{abstract}
Resumen-A pesar del notable esfuerzo llevado a cabo por la comunidad científica en el ámbito de la tecnología LTE, aún no existe una metodología globalmente aceptada para analizar este tipo de redes. Igualmente, no se conoce una única solución que responda a todos los requisitos que se pueden plantear a la hora de acometer su análisis, de modo que se emplean diferentes herramientas y soluciones, cada una con sus ventajas e inconvenientes. Una de las limitaciones más importantes es la dificultad para evaluar escenarios con un número elevado de elementos de red, lo que podría reflejar la situación de redes heterogéneas (HetNets). En otros casos, no se presta mucha atención a las características del nivel de servicio, suponiendo habitualmente que el sistema está saturado (full-buffer). Este trabajo presenta un entorno de simulación flexible y genérico (GWNSyM - Generic Wireless Network System Modeler), que permite el despliegue de escenarios complejos, y el análisis de diferentes técnicas y soluciones de gestión, así como nuevas arquitecturas de red. La herramienta se valida analizando una red muy heterogénea, con un elevado número de usuarios. Sobre este escenario se han analizado diferentes técnicas de acceso, incluyendo DUDe (Downlink-Uplink Decoupling), que plantea un cambio sustancial frente a las soluciones tradicionales de selección de acceso.
\end{abstract}

Palabras Clave-Network Modeling, Simulation, LTE/LTE-A, DUDe

\section{INTRODUCCIÓN}

De acuerdo a las previsiones actuales [1], la demanda de tráfico en redes móviles inalámbricas se incrementará de forma notable en los próximos años. Esto es debido, entre otras razones, a la consolidación de servicios de gran capacidad, tales como el streaming de vídeo o los juegos online, que compartirán los recursos de las redes con otros más tradicionales, como la navegación web o las descargas de ficheros.

Aunque a día de hoy las tecnologías 4G no están totalmente consolidadas, la comunidad investigadora ya está dirigiendo sus esfuerzos a la definición de las bases de la $5 \mathrm{G}$, que daría soporte a la previsible heterogeneidad de servicios. Así, se prevé que en el futuro coexistan redes de diferentes tecnologías, y que la cooperación entre ellas se lleve a cabo de manera natural. Por ejemplo, se espera que las estrategias de densificación mediante small-cells jueguen un papel muy importante en los próximos años [2], ya que pueden proporcionar un importante aumento de la capacidad. Otras técnicas que se han incluido recientemente en las especificaciones del 3GPP son las referidas a la cooperación entre elementos de red, o técnicas Cooperative Multi-Point (CoMP), o el desacoplamiento de los enlaces ascendente y descendente de las conexiones [3], o Downlink-Uplink Decoupling (DUDe).

Además de las soluciones que se sitúan en las capas inferiores de las redes celulares (gestión de recursos), en las capas superiores las técnicas de virtualización [4], Network Function Virtualization (NFV) y Software Defined Networks (SDN), se presentan como elementos clave de los despliegues de red en los próximos años [5].

A pesar de los claros avances que proporcionan estas soluciones, también originan nuevas problemáticas, que requieren un estudio y análisis apropiados. Para ello, la comunidad investigadora se centra habitualmente en escenarios específicos y casos de uso concretos, extraídos del amplio abanico de posibilidades que aparecen a raíz de los nuevos conceptos de red.

Uno de los primeros problemas a la hora de comenzar un análisis concreto es la elección de la herramienta, o conjunto de herramientas, que pueden ser usadas a fin de llevar a cabo un estudio adecuado, en función del nivel de abstracción requerido. Las plataformas de simulación juegan un papel fundamental, debido a su versatilidad y coste. En este sentido, existen básicamente tres alternativas principales: (1) simuladores a nivel de enlace, (2) a nivel de sistema, o (3) simuladores de red, que permiten acometer análisis más detallados. La primera opción se centra habitualmente en el último salto (inalámbrico) de la comunicación, y facilita la evaluación de técnicas de enlace, como la estimación de canal, técnicas MultipleInput Multiple-Output (MIMO) o soluciones relacionadas 
con el Adaptive Modulation and Coding (AMC). Dentro de este grupo Vienna LTE Simulator [6] destaca como una de las soluciones mayoritariamente adoptadas. La segunda alternativa permite una mayor flexibilidad, aunque como contrapartida suele requerir alguna simplificación, con la consiguiente pérdida de precisión. La mayoría de los simuladores a nivel de sistema se basan en desarrollos propietarios (normalmente basados en MATLAB), aunque en algunos casos se usan algunas de las pocas herramientas específicas disponibles, donde nuevamente destaca el simulador LTE Vienna [6]. Finalmente, en el tercer grupo existen varias plataformas, aunque la que seguramente está recibiendo más atención últimamente es ns-3 [7], y su extensión LTE-EPC Network Simulator (LENA) [8], que se encuentra en constante evolución. En el caso de simuladores de red, la mayor limitación son los tiempos para realizar los análisis, que se debe al alto grado de detalle de su implementación y modelos.

La Tabla I proporciona una comparativa detallada de las tres alternativas identificadas, en base a algunas de sus principales características. Una de las limitaciones que comparten las soluciones existentes es la dificultad para incluir o modelar nuevas técnicas y modelos de red, ya que su implementación suele ser bastante rígida, habitualmente centrada en escenarios concretos.

En base a las características de las soluciones de simulación existentes, surge la cuestión recurrente de qué herramienta se debería usar, de acuerdo al escenario y topología de red de interés, ya que no existe una solución que sea la idónea para todos los casos. El simulador Vienna LTE [6] está implementado en Matlab, y se centra en las capas inferiores, por lo que normalmente no es capaz de reflejar de manera apropiada diferentes patrones de servicio, adoptando habitualmente modelos de saturación o full-buffer. Por otro lado, los tiempos de simulación son bastante elevados, de modo que los análisis normalmente no cubren periodos de tiempo prolongados. Como consecuencia, en muchas ocasiones se opta por desarrollos propietarios, lo que requiere invertir un tiempo elevado en su desarrollo. Además, dado que se trata de soluciones ad-hoc, es complicado replicar los experimentos, así como integrarlos en otros entornos.

A fin de proporcionar una mejor respuesta, en este trabajo se presenta Generic Wireless Network System Modeler (GWNSyM), una plataforma flexible para la simulación de sistemas complejos. Esta herramienta se ha diseñado de modo genérico, para que sea fácilmente extensible con nuevas funcionalidades o soluciones de red. En este trabajo se presentan los principales aspectos tanto del diseño de la herramienta como de su implementación. Además, a modo de prueba de concepto, se presentará el análisis de diferentes técnicas de selección de acceso, haciendo uso de la herramienta.

El resto del artículo se estructura como sigue: en la Sección II se describe la funcionalidad básica de la herramienta, haciendo especial hincapié en los aspectos más relevantes de su implementación. A continuación, la Sección III presenta el escenario que se va a eva- luar, los modelos implementados, así como los resultados obtenidos. Finalmente, la Sección IV enumera las principales conclusiones de este trabajo, así como las lineas futuras de investigación que surgen al aprovechar las posibilidades ofrecidas por la herramienta.

\section{METODOLOGÍA DE SIMULACIÓN}

Como se ha comentado anteriormente, el objetivo principal de GWNSyM es el de proveer una serie de abstracciones que faciliten el modelado de redes para su posterior simulación. En lo que respecta a la metodología de análisis, se optó por una simulación basada en fotografías, o snapshots, del sistema, en contrapartida a otros modelos basados en eventos. De este modo, cada fotografía representa un instante discreto de tiempo, en el que se aplican los modelos implementados sobre los elementos de red pertinentes y en un orden establecido. Además, el estado resultante de una fotografía es usado para alimentar la siguiente, de modo que se puede capturar la memoria del sistema, lo que es especialmente importante para analizar la evolución de servicios.

El entorno de simulación GWNSyM ha sido implementado como un conjunto de librerías en $\mathrm{C}++$, teniendo como uno de sus objetivos principales la re-utilización de código, que puede darse en dos sentidos: (1) código generado en el entorno GWNSyM se pueda re-usar en otros entornos, y (2) código existente pudiera integrarse dentro del simulador. Para el primer caso, se decidió no imponer restricciones de herencias a las clases $\mathrm{C}++$ que implementan las diferentes entidades y modelos del simulador, sino que se estableció un mínimo interfaz que asegura la interacción de elementos GWNSyM. De forma resumida, por medio de técnicas de meta-programación se asegura que la compatibilidad entre los modelos implementados (por ejemplo, propagación o selección de acceso) y los elementos de red a los que se aplican, sin requerir ningún tipo de implementación (jerarquía, namespaces, etc.) específica, fomentando, de este modo, la separación de los modelos del sistema en el que se ejecutan. Del mismo modo, se ha añadido una funcionalidad de wrapper, que permite la integración de código existente dentro del entorno de simulación, dotándolo del interfaz requerido.

A continuación se explicará con más detalle los aspectos fundamentales de la simulación con GWNSyM, tanto en lo que se refiere a los elementos de simulación como al flujo de experimentación.

\section{A. Elementos de simulación}

Con el objetivo de no restringir el comportamiento de la herramienta de simulación a ninguna tecnología ni sistema en particular, se han definido dos elementos básicos que constituyen los escenarios de simulación GWNSyM: los Tipos representan elementos de red, mientras que las Acciones implementan modelos que se aplican sobre los Tipos.

Los Tipos definen la estructura de un elemento de red de forma general, junto con una configuración concreta. En este sentido, un elemento puede abarcar desde dispositivos 
Tabla I: Análisis de características de alternativas de simulación para redes inalámbricas (LTE). Una clasificación subjetiva se otorga a los parámetros, de forma que en círculos rellanos significa bueno, mientras que los vacíos indican pobre rendimiento en ese parámetros

\begin{tabular}{|c|c|c|c|c|}
\hline & $\begin{array}{c}\text { Parámetro } \\
\text { Descripción de la } \\
\text { característica de simulación } \\
\text { que se necesita soportar }\end{array}$ & $\begin{array}{l}\text { Simulación a nivel de enlace } \\
\text { Modelado detallado de capas } \\
\text { inferiores, lo que dificulta analizar } \\
\text { escenarios con mas de un par } \\
\text { fuente/destino }\end{array}$ & $\begin{array}{c}\text { Simulación a nivel de sistema } \\
\text { La mayoría de la literatura usa } \\
\text { Matlab para realizar análisis. Vienna } \\
\text { LTE Simulator es uno de los } \\
\text { ejemplos más significativos }\end{array}$ & $\begin{array}{c}\text { Simulación de red } \\
\text { ns-3, junto con la extensión LENA es } \\
\text { una de las alternativas más } \\
\text { relevantes }\end{array}$ \\
\hline \multirow{3}{*}{ 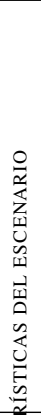 } & $\begin{array}{l}\text { Complejidad del escenario: \# } \\
\text { de usuarios y estaciones base }\end{array}$ & $\begin{array}{l}\text { Debido al gran nivel de detalle de } \\
\text { estas herramientas, el número de ele- } \\
\text { mentos el bastante bajo, normalmente } \\
\text { un elemento de acceso y un conjunto } \\
\text { de usuarios[9] }\end{array}$ & $\begin{array}{l}\text { Se suelen asumir alsgunas sim- } \\
\text { plificaciones, de modo que el número } \\
\text { de elementos suele ser mayor }\end{array}$ & $\begin{array}{l}\text { (1) El tiempo de simulación } \\
\text { requerido para analizar escenarios } \\
\text { grandes es normalmente } \\
\text { inaceptable [10], posibles alternativas } \\
\text { usando paralelización [11] }\end{array}$ \\
\hline & $\begin{array}{l}\text { Dimensión temporal: tiempo } \\
\text { que puede ser simulado y } \\
\text { posibilidad de estudiar la } \\
\text { evolución de servicios }\end{array}$ & $\begin{array}{l}(5 \text { Debido a la carga computa- } \\
\text { cional [12], el tiempo simulado es } \\
\text { bastante reducido, sin necesidad de } \\
\text { mantener evolución de servicios }\end{array}$ & $\begin{array}{l}\text { El uso de entornos de desarrollo } \\
\text { pesado (Matlab) normalmente impide } \\
\text { tiempos largos de simulación }\end{array}$ & $\begin{array}{l}\text { Normalmente se considera la } \\
\text { evolución de servicios, sin embargo, el } \\
\text { tiempo de cómputo para simulaciones } \\
\text { largas es muy elevado }\end{array}$ \\
\hline & $\begin{array}{l}\text { Precisión: grado de precisión } \\
\quad \text { de los modelos usados }\end{array}$ & $\begin{array}{l}\text { El modelado detallado de las ca- } \\
\text { pas inferiores es su principal objetivo, } \\
\text { por lo que la precisión es muy alta }\end{array}$ & $\begin{array}{l}\text { Se asumen algunas simpli- } \\
\text { ficaciones aunque implementaciones } \\
\text { disponibles siguen las especificaciones } \\
\text { del 3GPP }\end{array}$ & $\begin{array}{l}\text { Aunque los modelos pueden ser } \\
\text { simplificados, la implementación de } \\
\text { los protocolos es bastante precisa }\end{array}$ \\
\hline 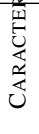 & $\begin{array}{l}\text { Cambio de arquitectura: } \\
\text { posibilidad de añadir y } \\
\text { soportar nuevos paradigmas } \\
\text { de red: SDN and NFV }\end{array}$ & $\begin{array}{l}\text { Como solución a nidel de enlace, } \\
\text { no se consideran problemáticas de ar- } \\
\text { quitectura }\end{array}$ & $\begin{array}{l}\text { Algunas de las posibilidades } \\
\text { de las nuevas funcionalidades de red } \\
\text { (tighter cooperation schemes) normal- } \\
\text { mente se pueden modelar }\end{array}$ & $\begin{array}{l}\text { Aunque la implementación puede } \\
\text { ser costosa, la integración de nuevas } \\
\text { opciones de arquitectura son normal- } \\
\text { mente posibles }\end{array}$ \\
\hline \multirow{2}{*}{ 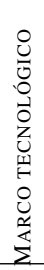 } & $\begin{array}{l}\text { Soporte de diferentes } \\
\text { tecnologías y } \\
\text { soluciones/técnicas }\end{array}$ & $\begin{array}{l}\text { (1) Se encuentran bastante limitadas } \\
\text { a las funcionalidades iniciales. La in- } \\
\text { tegración de diferentes tecnologías es } \\
\text { normalmente compleja }\end{array}$ & $\begin{array}{l}\text { Normalmente tienen flexibilidad } \\
\text { para incorporar nuevas técnicas debido } \\
\text { a las simplificaciones de capas inferi- } \\
\text { ores }\end{array}$ & $\begin{array}{l}\text { Las plataformas de simulación } \\
\text { de redes son bastante flexibles, y per- } \\
\text { miten la integración de diferentes tec- } \\
\text { nologías y técnicas nuevas }\end{array}$ \\
\hline & $\begin{array}{l}\text { Modelado de servicios. Si se } \\
\text { asume condiciones de } \\
\text { saturación o carga constante }\end{array}$ & $\begin{array}{l}\text { Do se presta mucha atención al } \\
\text { modelado de servicios, normalmente } \\
\text { se centra en cómo los paquetes llegan } \\
\text { a su destino en las capas inferiores }\end{array}$ & $\begin{array}{l}\text { (1) Presentan caracterización básica } \\
\text { de servicios, aunque normalmente } \\
\text { se asume carga constante o full- } \\
\text { buffer }[13]\end{array}$ & $\begin{array}{l}\text { Modelado de servicios relativa- } \\
\text { mente avanzado. Permite incluso el } \\
\text { uso de aplicaciones y servicios reales }\end{array}$ \\
\hline \multirow{2}{*}{$\begin{array}{l}n \\
0 \\
0 \\
0 \\
\text { w } \\
0 \\
0 \\
4 \\
0 \\
0 \\
0 \\
0 \\
0\end{array}$} & $\begin{array}{l}\text { Propósito específico Vs. } \\
\text { genérico y curva de } \\
\text { aprendizaje }\end{array}$ & $\begin{array}{l}\text { Como su ámbito de aplicación } \\
\text { está bastante delimitado, la curva de } \\
\text { aprendizaje es relativamente corta }\end{array}$ & $\begin{array}{l}\text { (1) Aunque más específicos que los } \\
\text { simuladores de red, no todos sus com- } \\
\text { ponentes son siempre de interés }\end{array}$ & $\begin{array}{l}(9 \text { Normalmente son grandes en- } \\
\text { tornos de propósito general, por lo } \\
\text { que requiere bastante tiempo de apren- } \\
\text { dizaje antes de poder realizar análisis }\end{array}$ \\
\hline & $\begin{array}{c}\text { Uso de metodologías } \\
\text { complementarias. Técnicas de } \\
\text { optimización }\end{array}$ & $\begin{array}{l}\text { (D) Normalmente se centran en } \\
\text { analizar el rendimiento de una técnica } \\
\text { concreta, y típicamente no buscan el } \\
\text { rendimiento óptimo }\end{array}$ & $\begin{array}{l}\text { (1) Aunque no está entre sus obje- } \\
\text { tivos principales, las técnicas de opti- } \\
\text { mización se pueden integrar }\end{array}$ & $\begin{array}{l}\text { (1) La arquitectura del simulador } \\
\text { ofrece una visión de conjunto, lo que } \\
\text { permitiría aplicar estrategias de opti- } \\
\text { mización global }\end{array}$ \\
\hline
\end{tabular}

de usuario a operadores, pasando por servicios o elementos virtuales. De acuerdo a su configuración, un Tipo puede agregar elementos de otro Tipo, de forma que se pueda definir la composición de cada elemento de red como una combinación de Tipos. La instanciación de elementos de un determinado Tipo define el conjunto de elementos correspondiente. A modo de ejemplo, la Figura 1 ilustra la creación de Tipos en GWNSyM para un caso genérico. Como se puede ver, el sustrato de los Tipos consiste en clases $\mathrm{C}++$ que, junto a una configuración concreta, da lugar a un Tipo. Así, la Figura 1 muestra cómo una misma clase $\mathrm{C}++C 1$ da lugar a dos Tipos ( $T 1$ y $T 2$ ), en función de la configuración que se le aplica. Además, de acuerdo a la configuración, las instancias de cada Tipo pueden dar lugar a diferentes agregaciones.

Las Acciones representan un modelo particular que se va a aplicar a uno o más conjuntos de Tipos. En general, las Acciones representan comportamientos del sistema en sentido amplio, pudiendo abarcar desde fenómenos físicos tales como modelos de propagación a políticas concretas, como selección de acceso. Cada Acción toma como parámetros uno o más conjuntos de Tipos, y se ejecutan de forma secuencial en cada fotografía del escenario. Por otro

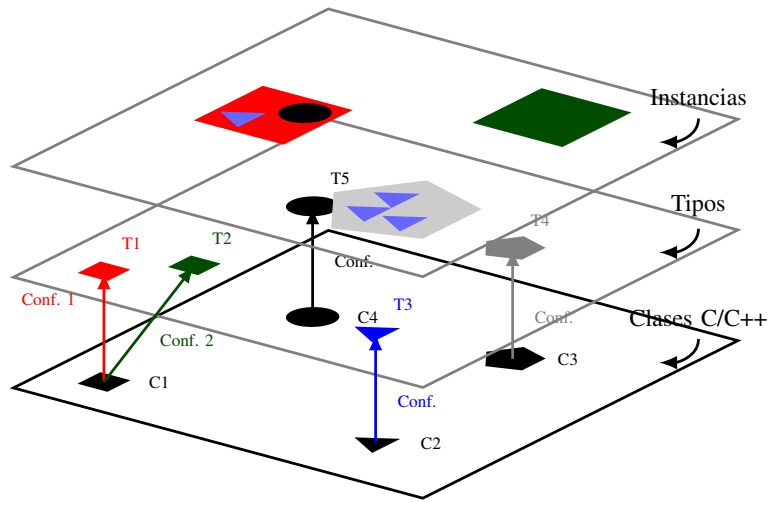

Fig. 1: Modelo de instanciación de GWNSyM

lado, hay ocasiones en las que una determinada Acción únicamente tiene sentido al inicio o final del experimento, como el despliegue de elementos estáticos, por ejemplo. Estos supuestos se han tenido en cuenta definiendo dos categorías de acciones, Pre-Acción y Post-Acción, que se ejecutan al principio y final del experimento, respectivamente. 


\section{B. Metodología de simulación}

Una instancia de simulación, o experimento, representa el análisis de un escenario concreto, sujeto a una configuración específica. Cada experimento contiene dos bucles: uno exterior y otro interior. El primero realiza interacciones fotografía a fotografía, actualizando el estado de la red de acuerdo al resultado de la iteración (fotografía) anterior. Por su parte, el segundo bucle se encarga de aplicar los modelos correspondientes a los elementos de red dentro de una fotografía.

El Algoritmo 1 ilustra la metodología general. Como primer paso, se definen los Tipos y se crean sus instancias, dando lugar a los conjuntos previamente mencionados, de acuerdo a su configuración. Seguidamente, una vez que todos los elementos del sistema han sido instanciados, se ejecutan las acciones definidas como Pre-Acciones. Llegado este punto, se inicia el primer bucle, línea 13, que itera sobre cada fotografía y el bucle interior, línea 14, que se encarga de ejecutar las acciones de forma secuencial. Tras finalizar ambos bucles, se aplican las Post-Acciones, normalmente encargadas de extraer resultados y generar trazas.

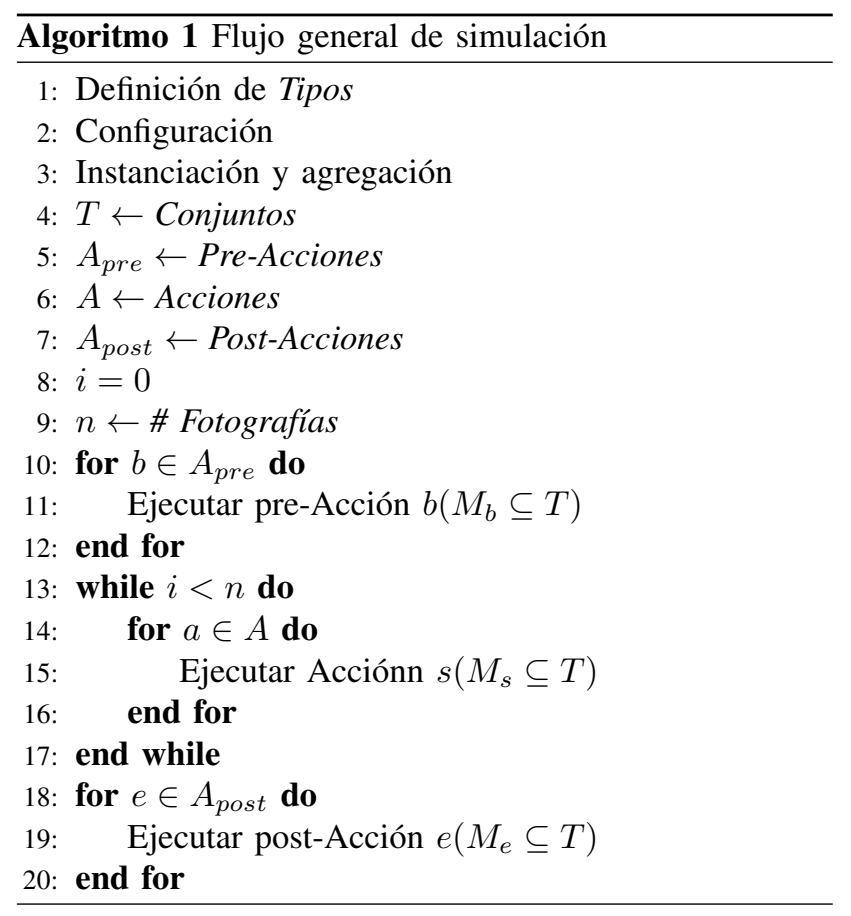

\section{Ejemplo de definición de un escenario}

A modo de ejemplo, la Figura 2 muestra los principales pasos de la definición de un escenario por medio de código. Como se puede observar en la Figura 2a, el primer paso consiste en el registro de tipos dentro del sistema. Los tipos consisten en dos objectos $\mathrm{C}++$ : el elemento de red y su configuración. Por ejemplo, el tipo USER se define en base a los objetos User y UserConf.

Antes de instanciar los elementos de cada tipo, el sistema comprueba aquellos que debe agregar, a través del nombre del tipo correspondiente. Como se muestra en la Figura2a, la configuración del tipo USER indica que

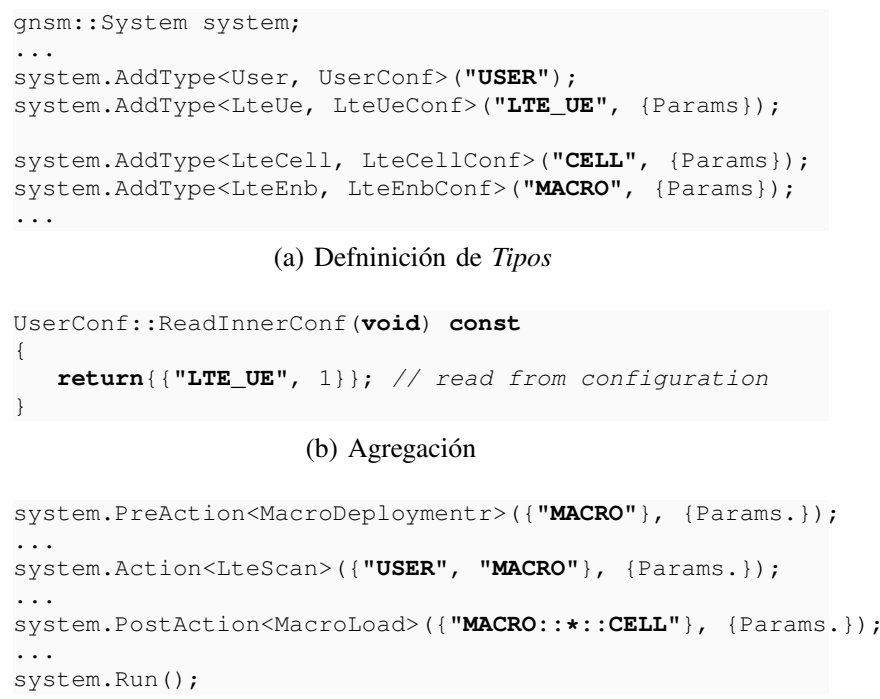

(a) Defninición de Tipos

(b) Agregación

system.PreAction<MacroDeploymentr>(\{"MACRO" \}, \{Params.\}); system.Action<LteScan> (\{"USER", "MACRO" \}, \{Params.\}); system.PostAction<MacroLoad> $\{$ "MACRO: : *::CELL" $\},\{$ Params. $\})$; syster system.Run();

(c) Acciones

Fig. 2: Ejemplo de definición de escenario

agrega una instancia del tipo LTE_UE, de modo que se instancia un elemento del tipo LTE_UE por cada elemento del tipo USER.

Una vez establecidos los tipos, se registran las acciones que definen el comportamiento del sistema, tal y como se muestra en la Figura 2c. Es importante destacar que se ha dotado al entorno de simulación de un módulo que permite la búsqueda de los elementos instanciados, de forma que el paso de conjuntos de elementos a las acciones se lleva a cabo de una manera muy flexible. Por ejemplo, MACRO: : : :CELL indicaría el conjunto de elementos CELL agregados en todas los elementos MACRO.

\section{ESCENARIO Y EVALUACIÓN}

A fin de validar el funcionamiento del entorno de simulación, esta sección presenta los principales resultados del estudio de diferentes técnicas de selección de acceso en un escenario LTE heterogéneo. En particular, se centra en la mejora en términos de potencia, y por tanto recursos, en el enlace ascendente, al aplicar diferentes políticas. $\mathrm{Si}$ bien la mayoría de los estudios se han centrado tradicionalmente en la gestión de recursos en el enlace descendente, la evolución de los servicios hace cada vez más necesario el prestar más atención a los recursos usados en la comunicación desde los usuarios a las estaciones base.

En las redes celulares un usuario se conecta habitualmente, tanto en el enlace ascendente como en el descendente, a aquella estación base de la que recibe una mejor calidad de la señal: en el caso concreto de redes LTE a la estación de la que recibe mayor Reference Signal Received Power (RSRP). Sin embargo, en entornos heterogéneos en los que co-existen estaciones base con un alto número de recursos y potencia de transmisión (macro-cells) con otras de capacidad más reducida (small cells), la selección de acceso basada en RSRP tiende a asociar a la mayor parte de usuarios con las que tienen una mayor potencia de transmisión, no permitiendo aprovechar el aumento de 
capacidad que aportan las small cells. Además, dado que el parámetro RSRP únicamente tiene en cuenta la calidad del enlace descendente, la potencia necesaria para transmitir desde los usuarios a las estaciones podría estar lejos de ser óptimo. Se debe tener en cuenta que la potencia recibida de las macro-cells puede ser mucho mayor que la de las small cells, incluso si la primera está situada notablemente más lejos del usuario.

A fin de establecer políticas de selección de acceso que aprovechen de forma más eficiente los recursos de las redes heterogéneas, se propuso el uso de técnicas Cell Range Extension (CRE) [14], que se basan en incrementar sintéticamente la potencia de la señal de referencia de las small-cells en un valor fijado (o bias), favoreciendo así las asociaciones a estos elementos de acceso. Además, teniendo en cuenta el incremento de los recursos consumidos en el enlace ascendente como consecuencia de la aparición de nuevos servicios, surge la necesidad de realizar una gestión más óptima de los recursos en el enlace ascendente. En este sentido, se ha propuesto recientemente una estrategia de selección de acceso que independiza los enlaces ascendente y descendente, conocida como Downlink Uplink Decouplig (DUDe) [15], de forma que cada usuario se asocie a aquel elemento que minimice los recursos necesarios para la transmisión. A continuación se presentarán los principales resultados del análisis de estas soluciones ante diferentes configuraciones.

El escenario que se ha utilizado durante el análisis está descrito en la Tabla II, que indica la topología del despliegue y los modelos de propagación implementados, que son los definidos en las recomendaciones del 3GPP. Se contemplan 7 celdas, desplegadas siguiendo un patrón hexagonal, y un número variable de small-cells en la zona de de las macro-cells central. Sobre este escenario se han desplegado varios usuarios, y se han aplicado las diferentes técnicas de selección: (1) RSRP, (2) técnicas CRE con diferentes valores de bias, y (3) DUDE.

Como se ha comentado anteriormente, este análisis se centra en el efecto que tienen las diferentes soluciones de selección de acceso sobre la potencia de transmisión necesaria en el enlace ascendente. Para ello se ha asumido que todos los usuarios requieren un valor de Signal to Noise plus Interference Ratio (SINR) fijo de $5 \mathrm{~dB}$, y se ha considerado que el sistema se encuentra en condiciones de saturación. Bajo estas premisas, se calcula la potencia de transmisión necesaria para que cada usuario alcance la SINR objetivo, de acuerdo al modelo de control de potencia en lazo abierto definido en la Ecuación 1 [16]:

$$
P_{t x}[d B m]=\min P_{\max }, P_{0}+10 \log _{10}\left(N_{R B}\right)+\alpha L
$$

donde $P_{t x}$ y $P_{\max }$ representan la potencia transmitida, y su valor máximo, respectivamente; $P_{0}$ indica la potencia que se transmite por unidad de recurso, $N_{R B}$ el número de recursos necesarios para transmitir, $L$ las pérdidas de propagación y $\alpha$ es el factor de compensación de propagación, que se ha fijado en 0.4 . Los parámetros $P_{\max }$ y $P_{0}$ se han fijado a 24 y $-80 \mathrm{dBm}$, respectivamente.
Tabla II: Configuración de la simulación

\begin{tabular}{|c|c|}
\hline \multicolumn{2}{|r|}{ FDD 2x20 MHz @ 2.1 GHz } \\
\hline Capa Macro & $\begin{array}{c}\text { ISD } 500 \mathrm{~m}, 7 \text { tri-sector sites } \\
\text { Max. tx. power } 46 \mathrm{dBm} \\
\text { Ganancia de antena } 15 \mathrm{dBi}, 15 \text { down-tilt }\end{array}$ \\
\hline Capa Small & $\begin{array}{c}\text { Despliegue aleatorio } \\
\text { Max. potencia transmisión } 37 \mathrm{dBm} \\
\text { Omni-antenna }\end{array}$ \\
\hline $\mathrm{UE}$ & $\begin{array}{c}\text { DL NF 7dB } \\
\text { Rx. Gain 7dB } \\
\text { Max. Potencia transmisión }\end{array}$ \\
\hline $\begin{array}{l}\text { Despliegue } \\
\text { LTE }\end{array}$ & L $(\mathrm{dB})$ función de la distancia $d[m]$ \\
\hline $\begin{array}{l}\text { Macro }_{\text {NLOS }} \\
\text { MacroLos }\end{array}$ & $\begin{array}{l}139.1033+39.0864 *\left(\log _{10}(d)-3\right) \\
36.2995+22 * \log _{10}(d) \quad \text { if } d<328.42 \\
40 * \log _{10}(d)-10.7953 \quad \text { if } d>328.42 \\
\end{array}$ \\
\hline $\begin{array}{l}\text { Small }_{\text {NLOS }} \\
\text { Small }_{\text {LOS }}\end{array}$ & $\begin{array}{l}145.48+37.5 *\left(\log _{10}(d)-3\right) \\
103.8+20.9 *\left(\log _{10}(d)-3\right) \\
\text { Probabilidad LOS función de la distancia } d[m\end{array}$ \\
\hline Macro & $\begin{array}{l}P_{L O S}=\min \left(\frac{18}{d}, 1\right) \cdot\left(1-e^{\frac{-d}{36}}\right)+e^{\frac{-d}{36}} \\
P_{L O S}=0.5-\min \left(0.5,5 \cdot e^{\frac{-156}{d}}\right) \\
\min \left(0.5,5 \cdot e^{\left.\left(\frac{-d}{30}\right)\right)}\right.\end{array}$ \\
\hline
\end{tabular}

Antes de analizar el comportamiento global de las diferentes estrategias de acceso, se ha estudiado la mejora potencial que se puede obtener en un escenario sencillo, en el que se sitúa una estación base de cada tipo, macro y small, con una separación de $1000 \mathrm{~m}$ entre ellas. Se asume que un usuario se irá desplazando en la línea recta que une ambos elementos de acceso, para caracterizar la potencia necesaria en el uplink en función de la posición en la que se encuentre. La Figura 3 muestra la potencia de transmisión en función de la distancia a la estación base macro. Cada punto representa el promedio de 1000 experimentos independientes, de manera que pueden haberse dado situaciones de conexión con cualquiera de las dos estaciones base, así como los percentiles 10 y 90 de dicha potencia de transmisión. Además, se indica el punto en el con mayor probabilidad se cambia de estación base. Como muestra la Figura 3, el uso de técnicas $C R E$ supone una notable ventaja en lo que se refiere a la utilización de la small-cell, de forma que cuanto mayor es el valor del bias, el traspaso de la macro a la small-cell ocurre antes. Siguiendo esta tendencia, se puede observar que el uso de DUDe aporta una mejora sustancial respecto a las otras alternativas, comprobándose que la variación de la potencia transmitida se reduce. De alguna manera, la técnica $D U D e$ equivale a usar $C R E$ con un valor de bias optimizado en cada momento.

A modo de resumen, la Figura 4 muestra los valores medio y máximo de potencia, utilizando las diferentes soluciones de selección de acceso. La figura muestra además de los valores globales (Max y Mean), los valores relativos a los casos en que se conecta a la estación base macro $\left(\operatorname{Max}_{M}\right.$ y $\left.\operatorname{Mean}_{M}\right)$ y small $\left(\operatorname{Max}_{S}\right.$ y Mean $\left.\operatorname{Me}_{S}\right)$. En general, se puede observar que a medida que aumenta el bias de CRE, y especialmente con el uso de DUDe, la potencia media necesaria al conectarse con la small-cell se incrementa, como consecuencia de que la conexión se establece con anterioridad. Sin embargo, también se observa que el hecho de potenciar el uso de las small-cells no conlleva una mejora sustancial en la potencia media 


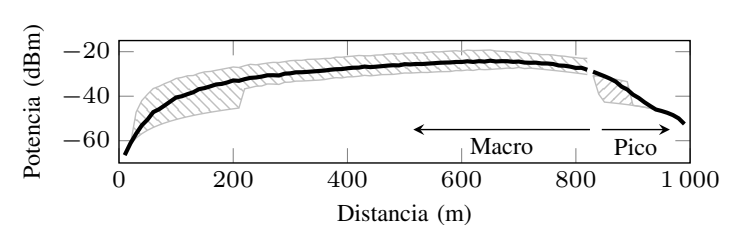

(a) RSRP

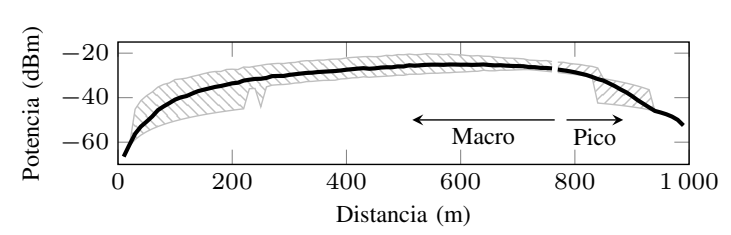

(b) CRE $6 d B$

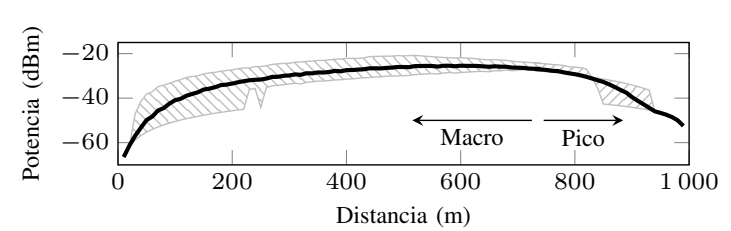

(c) CRE $9 d B$

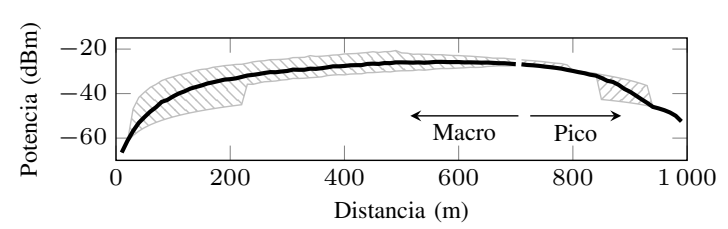

(d) CRE $12 d B$

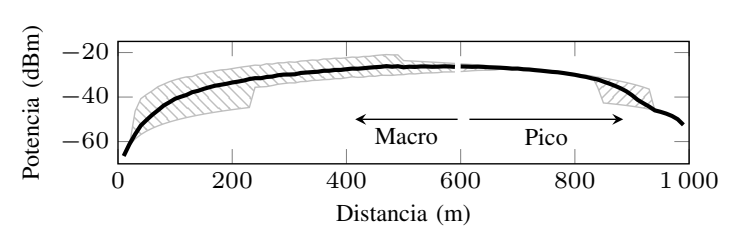

(e) DUDe

Fig. 3: Potencia media de transmisión en el enlace descendente a diferentes distancias de las estaciones base. La superficie sombreada delimita los percentiles del 10 y $90 \%$

transmitida, que se mantiene bastante constante para las diferentes estrategias de selección de acceso.

Finalmente se ha estudiado el rendimiento global de las diferentes técnicas en el escenario descrito en la Tabla II. Bajo la zona de cobertura de cada una de las macro-cell se ha desplegado un número creciente de small-cells, y se ha evaluado el comportamiento global de 6000 conexiones. La Figura 5 muestra la potencia media transmitida por cada una de las asociaciones usando las diferentes soluciones de selección de acceso, y para diferentes densidades de small-cells. Como se puede observar, al incrementar la presencia de small-cells se reduce notablemente la potencia necesaria para transmitir, siendo mucho menos relevante el impacto de las diferentes soluciones de selección de acceso. Además, la Figura 6 presenta la probabilidad de realizar una asociación con una macro-cell ante diferentes densidades de small-cells.

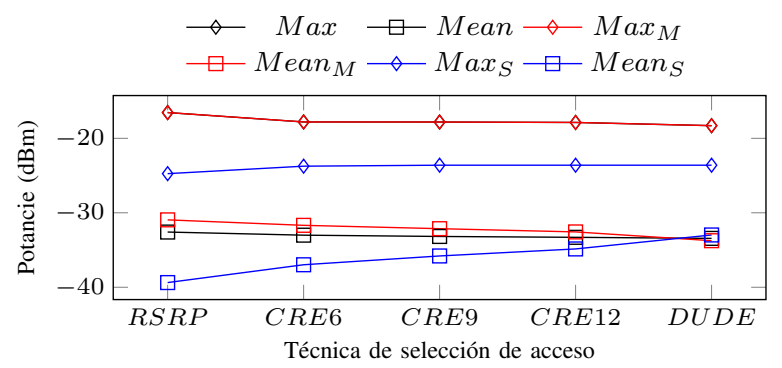

Fig. 4: Resumen de la potencia transmitida en las diferentes configuraciones. El eje de abscisas indica la técnica de acceso

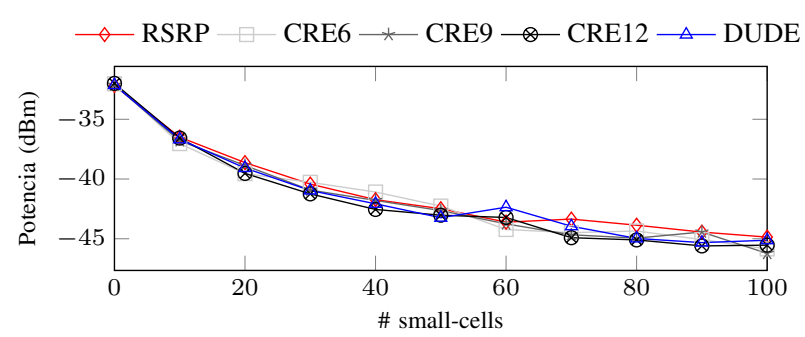

Fig. 5: Potencia media de transmisión ante diferente número de small-cells desplegadas

En este caso, sí se puede observar una diferencia notable para las diferentes técnicas de acceso utilizadas, de forma que $D U D e$ consigue incrementar considerablemente las conexiones con las small-cells, que incluso se llegan a duplicar, si se comparan con RSRP. Como es de esperar, esta diferencia se mitiga a medida que la densidad de small-cells aumenta.

\section{CONCLUSIONES}

Aunque la eclosión de las nuevas tecnologías ha ampliado de manera considerable las posibilidades de las redes celulares, su estudio se ha vuelto notablemente más complejo. En este sentido, la comunidad investigadora no se limita a analizar nuevas técnicas y soluciones que mejoren la calidad de los servicios, sino que es necesario evaluar las nuevas tendencias que afectan a las redes desde un punto de vista de su arquitectura. Como consecuencia, en este trabajo se ha argumentado que no existe una metodología o entorno de análisis de-facto. Así, aunque

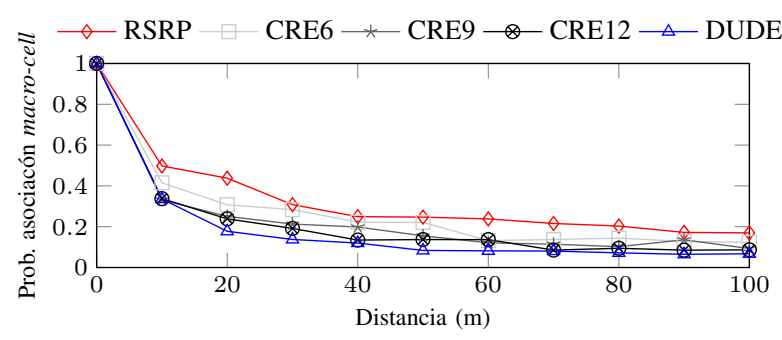

Fig. 6: Probabilidad media de asociación con la macro-cell. El eje de abscisas indica el número de small-cells desplegadas 
existen diversas alternativas, cada una de ellas presenta limitaciones, especialmente desde el punto de vista del impacto de los servicios (y los patrones de tráfico asociados) y de la cada vez mayor complejidad de las topologías.

Con el objetivo de dar respuesta a estas limitaciones, en este trabajo se ha presentado un entorno de simulación alternativo, Generic Wireless Network System ModelerGWNSyM. Su flexbilidad permite la definición de diferentes tipos de escenarios, que pueden analizarse de manera relativamente rápida y sencilla. Esta herramienta ha sido validada mediante el estudio de diferentes técnicas de selección de acceso, sobre un escenario heterogéneo. El análisis se ha centrado en cómo las diferentes técnicas explotan las capacidades adicionales de las small-cells y cómo esto afecta a la potencia transmitida en el enlace ascendente, desde el usuario a la estación base. De acuerdo a los resultados obtenidos, las nuevas técnicas, principalmente $D U D e$, son capaces de fomentar las conexiones con las estaciones base de menor capacidad. Sin embargo, mientras que la densificación de la red permite conseguir reducciones notables de la potencia transmitida, no se ha puesto de manifiesto un gran impacto de las diferentes técnicas.

Dada la flexibilidad del entorno de simulación presentado, se pretende aprovecharlo en diferentes ámbitos. En concreto, y en relación al análisis inicial presentado en este trabajo, en el futuro se pretende mejorar el modelado del escenario, teniendo en cuenta la evolución de los servicios, haciendo uso de un patrón de tráfico más acorde con los utilizados en la realidad. Además, se hará uso de herramientas que permitan establecer límites teóricos al rendimiento de las diferentes técnicas de selección, para lo que se integrarán algoritmos de optimización, tal y como ya se ha hecho previamente [17].

\section{AGRADECIMIENTOS}

Los autores agradecen la financiación del Gobierno de España (Ministerio de Economía y Competitividad, Fondo Europeo de Desarrollo Regional, FEDER) de este trabajo a través del proyecto ADVICE, Dynamic provisioning of connectivity in high density $5 \mathrm{G}$ wireless scenarios (TEC2015- 71329-C2-1-R).

\section{REFERENCIAS}

[1] C. and/or its affiliates, "Cisco visual networking index: Global mobile data traffic forecast update, 2015-2020 white paper," Feb. 2016. [Online]. Available: http://www.cisco.com/c/en/us/ solutions/collateral/service-provider/visual-networking-index-vni/ mobile-white-paper-c11-520862.html

[2] N. Bhushan, J. Li, D. Malladi, R. Gilmore, D. Brenner, A. Damnjanovic, R. Sukhavasi, C. Patel, and S. Geirhofer, "Network densification: the dominant theme for wireless evolution into $5 \mathrm{~g}$," IEEE Communications Magazine, vol. 52, no. 2, pp. 82-89, February 2014

[3] F. Boccardi, R. W. Heath, A. Lozano, T. L. Marzetta, and P. Popovski, "Five disruptive technology directions for $5 \mathrm{~g}$," IEEE Communications Magazine, vol. 52, no. 2, pp. 74-80, February 2014.

[4] M. Peng, Y. Li, Z. Zhao, and C. Wang, "System architecture and key technologies for $5 \mathrm{~g}$ heterogeneous cloud radio access networks," IEEE Network, vol. 29, no. 2, pp. 6-14, March 2015.

[5] M. Agiwal, A. Roy, and N. Saxena, "Next generation $5 \mathrm{~g}$ wireless networks: A comprehensive survey," IEEE Communications Sur veys Tutorials, vol. PP, no. 99, pp. 1-1, 2016.
[6] C. Mehlführer, J. Colom Ikuno, M. Šimko, S. Schwarz, M. Wrulich, and M. Rupp, "The vienna lte simulators - enabling reproducibility in wireless communications research," EURASIP Journal on Advances in Signal Processing, vol. 2011, no. 1, pp. 1-14, 2011. [Online]. Available: http://dx.doi.org/10.1186/1687-6180-2011-29

[7] "The ns-3 network simulator," http://www.nsnam.org/.

[8] G. Piro, N. Baldo, and M. Miozzo, "An lte module for the ns-3 network simulator," in Proceedings of the 4th International ICST Conference on Simulation Tools and Techniques, ser. SIMUTools '11. ICST, Brussels, Belgium, Belgium: ICST (Institute for Computer Sciences, Social-Informatics and Telecommunications Engineering), 2011, pp. 415-422. [Online]. Available: http://dl.acm.org/citation.cfm?id=2151054.2151129

[9] C. Schneider and R. S. Thomä, "Evaluation of lte link-level performance with closed loop spatial multiplexing in a realistic urban macro environment," in Antennas and Propagation (EUCAP), 2012 6th European Conference on, March 2012, pp. 2725-2729.

[10] R. M. Fujimoto, K. Perumalla, A. Park, H. Wu, M. H. Ammar, and G. F. Riley, "Large-scale network simulation: how big? how fast?" in Modeling, Analysis and Simulation of Computer Telecommunications Systems, 2003. MASCOTS 2003. 11th IEEE/ACM International Symposium on, Oct 2003, pp. 116-123.

[11] J. Pelkey and G. Riley, "Distributed simulation with mpi in ns-3," in Proceedings of the 4th International ICST Conference on Simulation Tools and Techniques, ser. SIMUTools '11. ICST, Brussels, Belgium, Belgium: ICST (Institute for Computer Sciences, Social-Informatics and Telecommunications Engineering), 2011, pp. 410-414. [Online]. Available: http://dl.acm.org/citation.cfm?id=2151054.2151128

[12] J. C. Ikuno, LTE Link- and System-Level Simulation. John Wiley \& Sons, Ltd, 2011, pp. 243-270. [Online]. Available: http://dx.doi.org/10.1002/9781119954705.ch11

[13] M. Taranetz, T. Blazek, T. Kropfreiter, M. K. Müller, S. Schwarz, and M. Rupp, "Runtime precoding: Enabling multipoint transmission in lte-advanced system-level simulations," IEEE Access, vol. 3, pp. 725-736, 2015.

[14] A. Damnjanovic, J. Montojo, Y. Wei, T. Ji, T. Luo, M. Vajapeyam, T. Yoo, O. Song, and D. Malladi, "A survey on 3 gpp heterogeneous networks," IEEE Wireless Communications, vol. 18, no. 3, pp. 1021, June 2011

[15] F. Boccardi, J. Andrews, H. Elshaer, M. Dohler, S. Parkvall, P. Popovski, and S. Singh, "Why to decouple the uplink and downlink in cellular networks and how to do it," IEEE Communications Magazine, vol. 54, no. 3, pp. 110-117, March 2016.

[16] S. Berger, B. Almeroth, V. Suryaprakash, P. Zanier, I. Viering, and G. Fettweis, "Dynamic range-aware uplink transmit power control in lte networks: Establishing an operational range for lte's open-loop transmit power control parameters (alpha, $\left.p_{0}\right)$, IEEE Wireless Communications Letters, vol. 3, no. 5, pp. 521-524, Oct 2014.

[17] L. Diez, G. P. Popescu, and R. Agüero, "A geometric programming solution for the mutual-interference model in hetnets," IEEE Communications Letters, vol. 20, no. 9, pp. 1876-1879, Sept 2016. 